\title{
Atitude e prática no consumo de frutas e hortaliças entre adolescentes em vulnerabilidade social
}

\author{
Attitude and practice about consumption of fruits and vegetables \\ among adolescents in social vulnerability
}

\section{Carla Rosane Paz Arruda Teo ${ }^{1}$, Roberta Lamonatto Taglietti², Francieli Baptista ${ }^{3}$, Vanessa Maria Meneghini ${ }^{3}$}

${ }^{1}$ Doutora em Ciência de Alimentos, Professora do Programa de Pós-Graduação em Ciências da Saúde da Universidade Comunitária da Região de Chapecó (UNOCHAPECÓ), Chapecó, SC; ${ }^{2}$ Nutricionista, Mestre em Ciências da Saúde pela UNOCHAPECÓ, Chapecó, SC; ${ }^{3}$ Nutricionistas graduadas pela UNOCHAPECÓ, Chapecó, SC.

\section{RESUMO}

Objetivos: Avaliar o comportamento alimentar para frutas e hortaliças de adolescentes em vulnerabilidade social.

Métodos: Estudo transversal com adolescentes assistidos por dois projetos sociais selecionados por amostragem de conveniência em Chapecó, estado de Santa Catarina. Aplicou-se Recordatório de 24 horas para analisar o consumo de frutas e hortaliças e um algoritmo específico para investigar a autopercepção sobre a qualidade do consumo e os estágios de mudança de atitude alimentar. Os dados foram analisados com o Statistical Package for Social Sciences v.17.0, por estatística descritiva e inferencial, adotando-se nível de significância de 5\%.

Resultados: Participaram do estudo todos os 120 adolescentes assistidos pelos projetos sociais selecionados, 59 meninos e 61 meninas, com

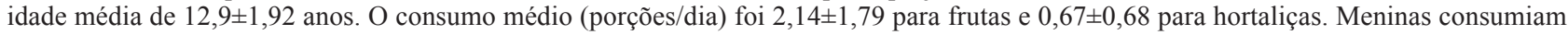
mais hortaliças $(\mathrm{p}=0,047) ; 52(43,3 \%)$ adolescentes consideravam ter consumo saudável de frutas, entretanto somente $24(46,2 \%)$ destes tinham consumo adequado; 61 (50,9\%) afirmaram consumo saudável de hortaliças, enquanto apenas dois (3,3\%) destes tinham consumo adequado. Os que consideravam ter consumo saudável consumiam mais frutas $(\mathrm{p}=0,001)$. Sobre o estágio de mudança, as meninas foram maioria em contemplação e decisão, e os meninos em pré-contemplação e ação ( $\mathrm{p}=0,014)$. O consumo de frutas apresentou correlação positiva significativa $\left(\mathrm{r}_{\mathrm{s}}=0,302 ; \mathrm{p}=0,001\right)$ com os estágios de mudança, sendo maior nos estágios mais avançados.

Conclusões: $O$ consumo de frutas e hortaliças foi inferior às recomendações, situação agravada pela autopercepção distorcida sobre a qualidade deste consumo e pela vulnerabilidade social. Os aspectos subjetivos do comportamento alimentar evidenciaram a predominância de adolescentes com consumo inadequado destes alimentos que não consideram a possibilidade de mudança de atitude alimentar.

DESCRITORES: CONSUMO DE ALIMENTOS; FRUTAS; HÁBITOS ALIMENTARES; NUTRIÇÃO DO ADOLESCENTE; VERDURAS.

\section{ABSTRACT}

Aims: To assess dietary behavior to fruit and vegetable intake of adolescents in social vulnerability.

Methods: Cross-sectional study with adolescents assisted by two social projects selected by convenience sampling in Chapeco, Santa Catarina state, Brazil. A 24-hour dietary recall was applied to analyze the consumption of fruits and vegetables, and a specific algorithm to investigate perception about the quality of consumption and the stages of change in eating attitude. Data were analyzed with Statistical Package for Social Sciences v.17.0, using descriptive and inferential statistics, with a significance level of 5\%.

Results: All the 120 adolescents assisted by the selected social projects, 59 boys and 61 girls, mean age $12.9 \pm 1.92$ years, participated in the study. Average consumption (servings/day) was $2.14 \pm 1.79$ for fruits and $0.67 \pm 0.68$ for vegetables. Girls consumed more vegetables ( $\mathrm{p}=0.047$ ); $43.3 \%$ of teens thought they have healthy intake of fruit, but only $46.2 \%$ of these had adequate intake; $50.9 \%$ said to have healthy vegetable consumption, while only $3.3 \%$ of these had adequate intake. Those who thought to have healthy intake, consumed more fruit ( $\mathrm{p}=0.001)$. On the stage of change, most girls were in contemplation and decision, and most boys in pre-contemplation and action ( $p=0.014)$. The consumption of fruits showed a significant positive correlation $\left(\mathrm{r}_{\mathrm{s}}=0.302, \mathrm{p}=0.001\right)$ with the stages of change, being higher in more advanced stages.

Conclusions: Consumption of fruits and vegetables was lower than recommendations, situation exacerbated by distorted perception about quality of consumption and by social vulnerability. Subjective aspects of feeding behavior revealed the predominance of adolescents with inadequate intake of these foods that do not consider the possibility of changing food attitude.

KEY WORDS: FOOD CONSUMPTION; FRUIT; FOOD HABITS; ADOLESCENT NUTRITION; VEGETABLES. 


\section{INTRODUÇÃO}

Para a Organização Mundial da Saúde (OMS), a adolescência é a fase compreendida entre os 10 e os 19 anos de idade. Conceitualmente, é uma fase de transição entre a infância e a idade adulta, marcada por transformações sociais, biológicas e psicológicas. ${ }^{1}$ Essas mudanças implicam o desenvolvimento de relações sociais, a realização de escolhas, o estabelecimento de valores, a adoção de diversos hábitos, entre eles os alimentares, e a consolidação de um estilo de vida. Os desequilíbrios alimentares nessa etapa da vida favorecem a ocorrência de desvios nutricionais, ${ }^{2}$ estando a alimentação do adolescente, na atualidade, em geral caracterizada por uma ingestão insuficiente de frutas e hortaliças, associada a um consumo excessivo de alimentos ricos em gorduras, açúcares e sódio. ${ }^{3}$

Os hábitos alimentares inadequados e o aumento do sedentarismo nessa fase estão diretamente relacionados com a obesidade, que marca o processo de transição nutricional constatado nas sociedades modernas, ${ }^{2}$ repercutindo em aumento da prevalência das doenças crônicas não transmissíveis na idade adulta. ${ }^{4,5}$ Nesse contexto, alguns alimentos (feijão, leite, frutas e hortaliças) têm sido adotados como marcadores de alimentação saudável pelo Ministério da Saúde no Brasil, ${ }^{6}$ devido ao fato de que seu consumo em nível adequado é reconhecido como protetor contra o desenvolvimento de doenças crônicas não transmissíveis. ${ }^{6,7}$

Alguns estudos têm evidenciado que a qualidade dos hábitos alimentares de adolescentes pode ter relação inversa com sua condição socioeconômica, ${ }^{3,8,9}$ e que alimentos básicos como feijão e arroz sejam mais consumidos por adolescentes de famílias economicamente menos favorecidas. ${ }^{10,11}$ Todavia, há que se considerar que adolescentes de baixa renda podem ter acesso limitado à quantidade suficiente de outros alimentos saudáveis, como frutas e hortaliças, devido ao custo que estes alimentos acrescem às despesas familiares. ${ }^{7}$ Assim, estando na condição de vulnerabilidade social, os alimentos mais preteridos pelas famílias costumam ser frutas e hortaliças, o que determina prejuízo à ingestão de micronutrientes, de fibras e de uma gama de fatores nutricionalmente essenciais e bioativos. Além disso, a baixa ingestão desses alimentos acaba compensada, muitas vezes, pela ingestão de outros alimentos, com altas concentrações de gordura, açúcar e sal. ${ }^{12}$

A primeira Pesquisa Nacional de Saúde do Escolar (PeNSE), realizada no ano de 2009, registrou uma prevalência de consumo regular (cinco ou mais dias na semana) de hortaliças de 34,3\% entre adolescentes de escolas privadas e de $30,4 \%$ entre os de escolas públicas. Para o consumo regular de frutas, foi relatada prevalência total de $31,5 \%$ naquela pesquisa. ${ }^{4} \mathrm{~A}$ segunda e mais recente edição da PeNSE, realizada em 2012, indicou que $43,4 \%$ dos adolescentes participantes referiram consumo regular de hortaliças e $30,2 \%$ de frutas. ${ }^{13}$

Os dados dessas pesquisas reforçam a importância de realizar estudos com diferentes abordagens sobre o consumo de alimentos protetores da saúde, a fim de subsidiar o planejamento e o desenvolvimento de intervenções para o enfrentamento do panorama epidemiológico atual no que tange aos agravos de origem alimentar e nutricional.

Diante do exposto, este estudo propôs avaliar o comportamento alimentar para frutas e hortaliças de adolescentes em condição de vulnerabilidade social, a partir de uma abordagem ampliada, incluindo aspectos quantitativos do consumo e aspectos subjetivos relativos às atitudes alimentares.

\section{MÉTODOS}

Foi realizado um estudo transversal, em que a amostra foi constituída por adolescentes assistidos por dois projetos sociais selecionados por conveniência, no município de Chapecó, em Santa Catarina. Os projetos sociais selecionados eram os únicos, à época, destinados à faixa etária de interesse para esta pesquisa no município, sendo que todos os adolescentes que os frequentavam foram esclarecidos, convidados e aceitaram participar do estudo. Os adolescentes frequentam os projetos diariamente no contraturno escolar, recebendo entre uma e duas refeições durante esse período.

Para a análise quantitativa do consumo de frutas e hortaliças foi aplicado o Recordatório 24 horas em entrevista individual, por pesquisador previamente capacitado. Para a avaliação de aspectos subjetivos da atitude alimentar foi utilizado um algoritmo previamente validado, ${ }^{14} \mathrm{a}$ fim de investigar a percepção dos adolescentes sobre a qualidade de seu consumo alimentar de frutas e hortaliças, e para identificar o estágio de mudança de atitude em relação ao consumo desses alimentos. $\mathrm{O}$ algoritmo contém quatro perguntas fechadas que abordam se o adolescente considera seu consumo do alimento em questão (frutas, hortaliças) saudável, há quanto tempo considera que seu consumo poderia ser classificado como saudável, se está considerando seriamente a possibilidade de aumentar seu consumo daquele alimento nos próximos meses, e qual o grau de confiança do adolescente quanto a tornar realidade esta possibilidade. 
O algoritmo foi aplicado de forma independente para cada um dos dois grupos alimentares de interesse, permitindo classificar os adolescentes em cinco estágios de mudança para frutas e para hortaliças. Os estágios de mudança considerados foram os de pré-contemplação, contemplação, decisão, ação e manutenção. O estágio de pré-contemplação indica uma fase em que a pessoa não tem a intenção de mudar a atitude de risco em um futuro próximo; contemplação é o estágio em que o indivíduo evidencia a intenção de mudança nos próximos seis meses; o estágio de decisão é aquele em que o indivíduo decide efetivamente alterar sua atitude em um período curto de tempo (geralmente o próximo mês); ação é o estágio em que o indivíduo apresenta uma mudança efetiva de atitude em um período recente (nos últimos seis meses); manutenção é o estágio em que o indivíduo mudou uma atitude e mantém essa mudança por um período igual ou superior a seis meses. ${ }^{15}$

Os dados foram analisados com o Statistical Package for the Social Sciences v.17.0, por meio de estatística descritiva (frequências absoluta e relativa, média, desvio padrão, mediana) e inferencial (teste de Tukey, teste de Mann-Whitney, qui-quadrado, teste exato de Fischer, correlação de Spearman), adotando-se nível de significância de 5\%.

Este estudo foi aprovado pelo Comitê de Ética em Pesquisa da Universidade Comunitária da Região de Chapecó - UNOCHAPECÓ (Parecer $n^{\circ} 117$ ), e todos os princípios éticos da pesquisa envolvendo seres humanos foram rigorosamente respeitados, incluindo assinatura do termo de consentimento livre e esclarecido pelos responsáveis legais dos adolescentes.

\section{RESULTADOS}

Participaram deste estudo 120 adolescentes, com idade média de 12,9 anos $( \pm 1,92)$, dos quais $61(50,8 \%)$ eram meninas. Destaca-se que, segundo o sexo, os participantes apresentaram diferença significativa apenas quanto às variáveis consumo de hortaliças (Tabela 1) e estágio de mudança da atitude alimentar para frutas (Tabela 2). O consumo de hortaliças, em número médio de porções diárias, foi maior entre as meninas $(0,79$ versus 0,54 nos meninos; $p=0,047$ ). Quanto aos estágios de mudança, as meninas foram maioria nos estágios de contemplação e decisão, e os meninos nos estágios de pré-contemplação e ação $(\mathrm{p}=0,014)$.

Tabela 1. Consumo médio e adequação do consumo de frutas e hortaliças dos adolescentes avaliados segundo o sexo.

\begin{tabular}{|c|c|c|c|c|}
\hline Variável & $\begin{array}{c}\text { Total } \\
(n=120)\end{array}$ & $\begin{array}{c}\text { Meninos } \\
(n=59)\end{array}$ & $\begin{array}{c}\text { Meninas } \\
(n=61)\end{array}$ & $p$ \\
\hline Consumo de frutas* (Média \pm DP) & $2,14 \pm 1,79$ & $2,31 \pm 1,98$ & $1,98 \pm 1,58$ & $0,327+$ \\
\hline Adequado -n (\%) & $40(33,3)$ & $20(33,9)$ & $20(32,8)$ & $0,897 \neq$ \\
\hline Não adequado - n (\%) & $80(66,7)$ & $39(66,1)$ & $41(67,2)$ & \\
\hline Consumo de hortaliças* (Média \pm DP) & $0,67 \pm 0,68$ & $0,54 \pm 0,68$ & $0,79 \pm 0,66$ & $0,047+$ \\
\hline Adequado - n (\%) & $2(1,7)$ & $1(1,7)$ & $1(1,6)$ & $0,744 \neq$ \\
\hline Não adequado - n (\%) & $118(98,3)$ & $58(98,3)$ & $60(98,4)$ & \\
\hline
\end{tabular}

DP: desvio padrão. *Adequado: ingestão $\geq 3$ porções/dia; não adequado: ingestão $<3$ porções/dia. ${ }^{+}$Valor obtido pelo teste de Mann-Whitney. ${ }^{\ddagger}$ Valor obtido pelo teste qui-quadrado de Pearson ou Exato de Fischer

Tabela 2. Estágio de mudança da atitude alimentar dos adolescentes avaliados para frutas e hortaliças segundo o sexo.

\begin{tabular}{|c|c|c|c|c|c|c|c|}
\hline \multirow[t]{2}{*}{ Estágio de Mudança } & \multicolumn{2}{|c|}{$\begin{array}{c}\text { Total } \\
(n=120)\end{array}$} & \multicolumn{2}{|c|}{$\begin{array}{c}\text { Meninos } \\
(n=59)\end{array}$} & \multicolumn{2}{|c|}{$\begin{array}{c}\text { Meninas } \\
(n=61)\end{array}$} & \multirow{2}{*}{$p$} \\
\hline & $\mathbf{n}$ & $(\%)$ & $\mathbf{n}$ & $(\%)$ & $\mathbf{n}$ & $(\%)$ & \\
\hline \multicolumn{8}{|l|}{ Atitude alimentar/frutas } \\
\hline Pré-contemplação & 33 & $(27,5)$ & 21 & $(35,6)$ & 12 & $(19,7)$ & \multirow{5}{*}{$0,014^{*}$} \\
\hline Contemplação & 19 & $(15,8)$ & 5 & $(8,5)$ & 14 & $(23,0)$ & \\
\hline Decisão & 16 & $(13,3)$ & 4 & $(6,8)$ & 12 & $(19,7)$ & \\
\hline Ação & 27 & $(22,5)$ & 17 & $(28,8)$ & 10 & $(16,4)$ & \\
\hline Manutenção & 25 & $(20,8)$ & 12 & $(20,3)$ & 13 & $(21,3)$ & \\
\hline \multicolumn{8}{|l|}{ Atitude alimentar/hortaliças } \\
\hline Pré-contemplação & 33 & $(27,5)$ & 18 & $(30,5)$ & 15 & $(24,6)$ & \multirow{5}{*}{$0,726^{*}$} \\
\hline Contemplação & 14 & $(11,7)$ & 7 & $(11,9)$ & 7 & $(11,5)$ & \\
\hline Decisão & 12 & $(10,0)$ & 6 & $(10,2)$ & 6 & $(9,8)$ & \\
\hline Ação & 32 & $(26,7)$ & 17 & $(28,8)$ & 15 & $(24,6)$ & \\
\hline Manutenção & 29 & $(24,2)$ & 11 & $(18,6)$ & 18 & $(29,5)$ & \\
\hline
\end{tabular}

* Valor obtido pelo teste qui-quadrado de Pearson ou Exato de Fischer. 
A avaliação da autopercepção dos adolescentes sobre a qualidade de seu consumo de frutas e hortaliças, derivada da pergunta do algoritmo que questionava se o adolescente considerava seu consumo saudável, foi comparada com os resultados do consumo relatado

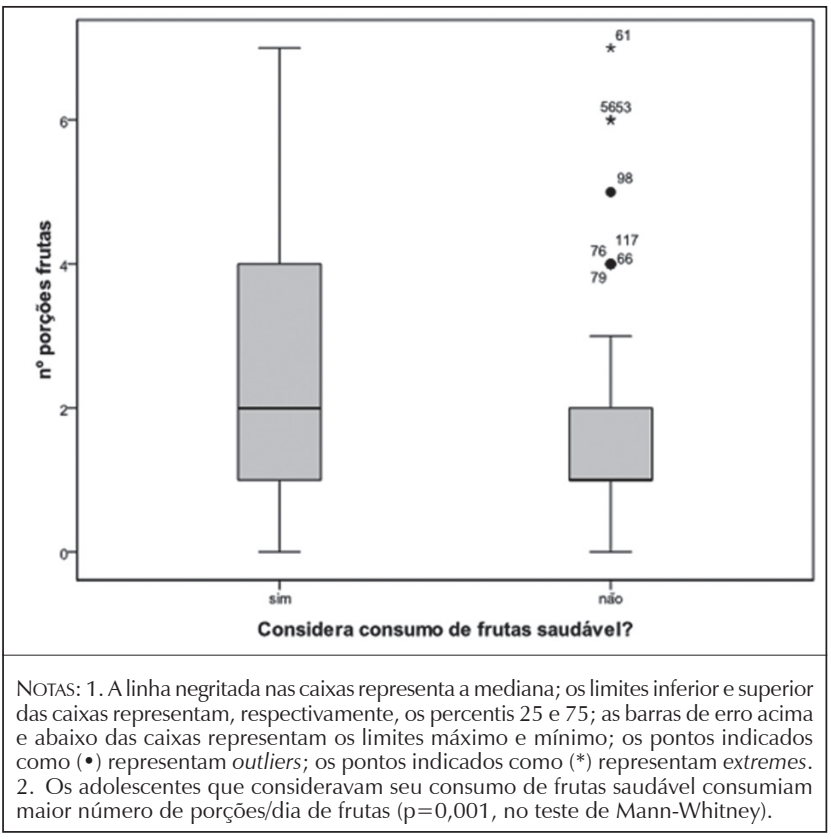

Figura 1. Consumo de frutas, em número de porções/dia, segundo a percepção de consumo saudável dos adolescentes avaliados.

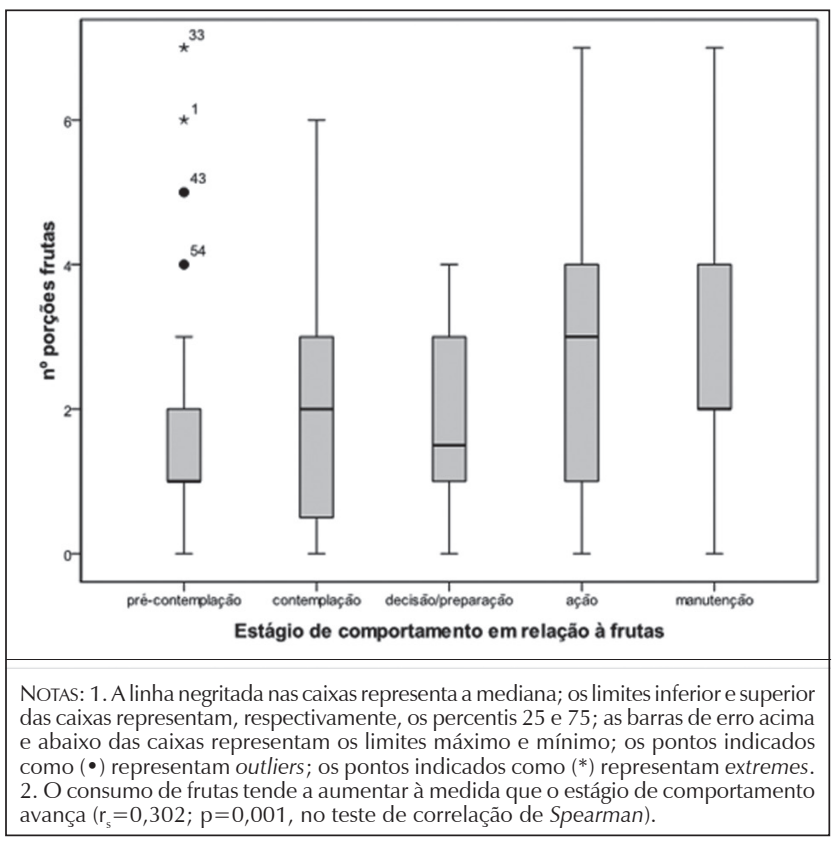

Figura 2. Correlação entre o consumo de frutas, em número de porções/dia, e o estágio de mudança de atitude alimentar para frutas dos adolescentes avaliados. na aplicação do Recordatório 24 horas. Esta análise evidenciou que $52(43,3 \%)$ adolescentes consideravam ter um consumo saudável de frutas, dentre os quais somente $24(46,2 \%)$ consumiam estes alimentos na quantidade diária adequada; para o consumo de hortaliças, $61(50,9 \%)$ adolescentes afirmaram ter um consumo saudável, dos quais apenas dois $(3,3 \%)$ apresentavam consumo adequado.

Os adolescentes que consideravam seu consumo de frutas como sendo saudável efetivamente consumiam mais frutas $(\mathrm{p}=0,001)$ (Figura 1). Para o consumo de hortaliças esta associação não foi significativa $(\mathrm{p}=0,133)$.

Quando analisado o consumo de frutas em relação com o estágio de mudança da atitude alimentar para frutas, foi observada correlação positiva significativa entre essas variáveis $\left(\mathrm{r}_{\mathrm{s}}=0,302 ; \mathrm{p}=0,001\right)$, sendo que o consumo de frutas apresentou tendência a aumentar à medida que o estágio de comportamento avançou (Figura 2). O mesmo não ocorreu com o consumo de hortaliças $\left(r_{s}=0,092 ; p=0,318\right)$.

A análise do consumo médio de frutas e hortaliças (em porções/dia), segundo o estágio de mudança, evidenciou que as maiores médias diárias de consumo foram relatadas pelos adolescentes que apresentaram atitude característica dos estágios de ação e manutenção (Tabela 3).

Tabela 3. Consumo médio de frutas e hortaliças dos adolescentes avaliados segundo o estágio de mudança de atitude alimentar.

\begin{tabular}{|lcc|}
\hline \multirow{2}{*}{ Estágio de Mudança } & \multicolumn{2}{c}{$\begin{array}{c}\text { Consumo médio (porções/dia) } \\
\text { Média } \pm \text { DP* }\end{array}$} \\
\cline { 2 - 3 } & \multicolumn{1}{c}{ Frutas } & Hortaliças \\
\hline Pré-contemplação & $1,58 \pm 1,70^{\mathrm{d}}$ & $0,61 \pm 0,61^{\mathrm{c}}$ \\
\hline Contemplação & $1,84 \pm 1,68^{\mathrm{b}}$ & $0,71 \pm 0,61^{\mathrm{bc}}$ \\
\hline Decisão & $1,69 \pm 1,25^{\mathrm{c}}$ & $0,25 \pm 0,45^{\mathrm{d}}$ \\
\hline Ação & $2,74 \pm 1,74^{\mathrm{a}}$ & $0,78 \pm 0,79^{\mathrm{a}}$ \\
\hline Manutenção & $2,76 \pm 2,05^{\mathrm{a}}$ & $0,76 \pm 0,69^{\mathrm{ab}}$ \\
\hline
\end{tabular}

* Comparação entre médias pelo teste Tukey.

Médias na mesma coluna acompanhadas de letras diferentes diferem significativamente $(p \leq 0,05)$.

\section{DISCUSSÃO}

Observou-se, entre os adolescentes avaliados, um consumo médio de frutas e hortaliças inferior à recomendação do Guia Alimentar para a População Brasileira, ${ }^{16}$ que é de três porções diárias de cada um destes grupos de alimentos. Cabe destacar, neste ponto, o consumo relatado de hortaliças, que esteve bastante abaixo desta recomendação. 
Os dados de consumo médio de frutas e hortaliças somados, identificados neste estudo, são compatíveis com a mais recente Pesquisa de Orçamento Familiar (POF 2008-2009) realizada no Brasil, que indicou a necessidade de ser pelo menos triplicado o consumo destes alimentos para que sejam atingidos níveis de adequação. ${ }^{17}$

Segundo a última PeNSE, ${ }^{13}$ a prevalência de consumo regular é de $43,4 \%$ para hortaliças e de $30,2 \%$ para frutas entre adolescentes brasileiros. Porém, a PeNSE utiliza o conceito de consumo regular, que está muito aquém do que seria suficiente para a prevenção das doenças crônicas que podem ser evitadas com adequado consumo de frutas e hortaliças, ${ }^{4}$ enquanto o presente estudo utiliza o conceito de adequação à recomendação, que é mais exigente.

Apesar da prevalência identificada de consumo inadequado de frutas e hortaliças, recorrentemente observada em estudos anteriores desenvolvidos com adolescentes, ${ }^{2,18-21}$ uma elevada proporção dos participantes deste estudo considerava saudável seu consumo desses alimentos. Todavia, dentre os que consideram saudável seu consumo, apenas cerca de metade relatou consumo adequado de frutas, e uma ínfima parcela referiu adequação para o de hortaliças, o que parece indicar que a noção de consumo saudável, para os adolescentes avaliados, não está atrelada à de adequação, ou que eles desconhecem as recomendações para o consumo destes alimentos. Há que se considerar também que a discrepância observada possa resultar de uma necessidade social do adolescente em parecer ter uma alimentação saudável. ${ }^{22}$

Na mesma linha, outra possível explicação para esta discrepância é o fato de os adolescentes observarem no cotidiano que seu consumo de frutas e hortaliças supera o de outras pessoas de seu círculo de convivência, passando a considerar que seu consumo seja saudável. Neste sentido, verificou-se que os adolescentes que referiram uma percepção de consumo saudável foram os que efetivamente relataram maior consumo diário de frutas, embora não necessariamente adequado. Esta observação não foi válida para o grupo das hortaliças.

Independentemente da causa, discrepâncias entre o consumo alimentar referido e a autopercepção de sua adequação podem contribuir para situar os adolescentes como grupo de risco, exigindo cuidado especial quanto à promoção de alimentação saudável, ${ }^{14}$ considerando que nesta etapa da vida as necessidades nutricionais são aumentadas devido ao estirão de crescimento e transformações corporais características da puberdade. ${ }^{23}$
Prosseguindo na análise, observou-se que os adolescentes estiveram concentrados no estágio de pré-contemplação em termos da atitude alimentar para frutas e hortaliças, seguido pelos estágios de ação e manutenção. A prevalência no estágio de précontemplação corrobora a importância da realização de ações educativas de caráter informativo voltadas ao incentivo do consumo de frutas e hortaliças, abordando sua importância para a saúde, principalmente na adolescência, uma vez que nesse estágio da vida os indivíduos, além de mais resistentes a mudanças alimentares, ainda carecem de informações que os tornem conscientes da necessidade de mudança para que passem a cogitar essa possibilidade..$^{14,21}$ Salienta-se que o fato dos adolescentes que participaram deste estudo estarem em condição de vulnerabilidade social pode levar a que tenham menor acesso a estas informações, ou que tenham prejudicada sua capacidade de aplicálas em suas práticas cotidianas.

Tomando-se em conjunto os estágios de ação e manutenção, a frequência de adolescentes supera a do estágio de pré-contemplação. Uma vez que a classificação nesses estágios avançados implica que os adolescentes tenham manifestado que consideram seu consumo saudável, devido à ordem de apresentação das perguntas no algoritmo utilizado, este resultado reforça a noção de uma percepção distorcida neste grupo quando são considerados os índices de consumo realmente adequado para esses alimentos.

Isso significa dizer que um número relevante de adolescentes demonstrou não perceber a inadequação do próprio consumo de frutas e hortaliças. Evidencia-se que, além de baixa percepção sobre a adequação do próprio consumo, há ainda um percentual relativamente elevado de adolescentes com consumo inadequado de frutas e hortaliças imobilizado nos estágios de pré-contemplação, ação e manutenção, sendo esta situação mais acentuada para hortaliças. Fato semelhante foi relatado por Toral et al., ${ }^{14}$ quando observaram que $33,8 \%$ e $44,5 \%$ dos adolescentes avaliados se situavam em estágio de ação e manutenção para frutas e para hortaliças, respectivamente, enquanto os índices de consumo adequado para esses alimentos eram de apenas $12,4 \%$ e $5,6 \%$.

Constatou-se, ainda, uma correlação positiva significativa entre o consumo e o estágio de mudança de atitude alimentar para frutas, havendo uma tendência de aumento do consumo destes alimentos na medida em que o estágio de atitude alimentar avança. No entanto, essa tendência não se repetiu para o consumo de hortaliças. Buscando compreender melhor a relação entre a atitude alimentar e o consumo 
de frutas e hortaliças, foi avaliado o consumo médio desses alimentos (em porções/dia) segundo o estágio de mudança, tendo sido observado que as maiores médias diárias de consumo foram relatadas pelos adolescentes que apresentaram atitude característica dos estágios de ação e manutenção.

Estas observações reforçam a hipótese de que o fato de alguns adolescentes apresentarem um consumo mais elevado dentro do grupo pode ser um dos elementos que os leva a assumir uma atitude de ação e manutenção, mesmo estando em um patamar de consumo ainda insuficiente. Outra consideração que pode ser feita a partir destes achados é a de que os adolescentes realmente tenham implementado alguma mudança em seu consumo de frutas e hortaliças em período recente ou que a estejam mantendo por seis meses ou mais, mas que essa mudança não tenha elevado o consumo ao nível de adequação, provavelmente devido à condição social vulnerável desses indivíduos, visto que estes são os alimentos mais preteridos por populações em risco social. ${ }^{12}$

Quando os dados foram analisados segundo o sexo dos adolescentes, apenas duas variáveis apresentaram diferença neste estudo: o consumo de hortaliças significativamente menor entre os meninos e o estágio de atitude alimentar para frutas, com os meninos sendo maioria nos estágios de pré-contemplação e ação, e as meninas nos estágios de contemplação e decisão.

Com relação às hortaliças, diversos estudos têm referido que o consumo desses alimentos tende a ser maior e mais frequente no sexo feminino, independentemente da faixa etária, em função do padrão estético contemporâneo. $\cdot^{18,24-27}$ Contudo, esta explicação se aplicaria, em teoria, também ao consumo de frutas, o que sugere que existem outros condicionantes para as diferenças no consumo de alimentos protetores da saúde entre os sexos.

No caso deste estudo, sugere-se que o consumo de hortaliças esteja mais restrito ao âmbito doméstico e, portanto, seja de acesso mais limitado, o que explicaria seu consumo médio extremamente baixo para o grupo como um todo, e menor entre os meninos. Já as frutas são mais frequentemente acessadas pelos adolescentes na alimentação escolar e nos projetos sociais, devido às características das refeições servidas nestes locais, de onde decorreria seu maior consumo médio e a não existência de diferenças entre os sexos.

Neste ponto, esclarece-se que todos os adolescentes deste estudo frequentam a escola, o que é pré-requisito para seu ingresso nos projetos sociais. $\mathrm{Na}$ alimentação escolar do município, são servidas frutas um mínimo de três vezes na semana. Já nos projetos sociais, os adolescentes recebem de uma a duas refeições diárias, todas incluindo a oferta de frutas. As hortaliças, por outro lado, são acessadas mais esporadicamente na alimentação escolar, e em apenas uma das refeições nos projetos sociais, mas não diariamente. Argumenta-se, neste contexto, que o acesso a hortaliças, para o grupo em estudo, seja mais dependente do ambiente familiar.

A constatação de maior frequência de meninos nos estágios de pré-contemplação e ação para o consumo de frutas, em princípio, indica que uma grande parcela dos meninos está situada em um estágio de maior resistência à mudança alimentar (pré-contemplação), ${ }^{14}$ seguido de outro que aponta para uma mudança alimentar em curso (ação), ${ }^{15}$ sendo que esta última observação se contrapõe a um consumo médio de frutas inferior à recomendação. No caso das meninas, os achados são mais coerentes, uma vez que as maiores frequências estiveram nos estágios de contemplação e decisão, mais favoráveis à mudança alimentar, ${ }^{15} \mathrm{de}$ forma condizente com o consumo médio de frutas identificado.

Ressalta-se a importância de que o planejamento das ações educativas considere a percepção e a atitude alimentar dos adolescentes, pois tem sido relatado que o não reconhecimento da inadequação das próprias práticas alimentares representa um obstáculo significativo ao sucesso de programas de educação nutricional, tendo em vista que, nesse caso, não há motivação para a mudança. ${ }^{14}$ Estes achados são importantes para o planejamento das ações de intervenção, pois permitem contextualizá-las em função da percepção, motivação e grau de confiança para mudar. ${ }^{28}$ Salienta-se também que qualificar a intervenção educativa dirigida a adolescentes pode contribuir para o enfrentamento dos principais agravos à saúde na atualidade, já que a adolescência é considerada uma etapa do curso da vida propícia para a consolidação de hábitos alimentares que, se adequados, poderão ter reflexos favoráveis na saúde em médio e longo prazo. ${ }^{29}$

$\mathrm{Na}$ literatura publicada, reconhece-se que um bom nível de conhecimento sobre alimentação saudável, mesmo que associado a um adequado acesso aos alimentos, não resulta necessariamente em comportamento alimentar saudável e adequado, especialmente entre adolescentes. ${ }^{20,30}$ Sugere-se que esta afirmação seja ainda mais válida em se tratando de adolescentes em condição de vulnerabilidade social, visto que seu acesso aos alimentos, principalmente às frutas e hortaliças, pode estar bastante comprometido. $^{12}$ 
Os achados deste estudo corroboram a inadequação quantitativa do consumo de frutas e hortaliças entre adolescentes. A avaliação de aspectos subjetivos relativos à atitude alimentar revelou autopercepção distorcida sobre a adequação deste consumo, havendo uma concentração de adolescentes com consumo inadequado imobilizado nos estágios iniciais (précontemplação), sem considerar a possibilidade de mudança, e nos finais (ação e manutenção), considerando a mudança já concretizada.
Estas observações podem contribuir para o planejamento de intervenções em educação nutricional, orientando ações informativas, de sensibilização, motivacionais, ou de apoio e reforço. Da mesma forma, destaca-se a importância de avaliar o comportamento alimentar a partir de abordagens ampliadas, que combinem aspectos quantitativos e subjetivos implicados no consumo, pelo fato destas abordagens constituírem, a um só tempo, elementos para o planejamento e indicadores de efetividade.

\section{REFERÊNCIAS}

1. World Health Organization (WHO). Pregnancy and Abortion in Adolescence. Report of a WHO Meeting. WHO Technical Report Series no. 583. Geneva: WHO; 1975.

2. Toral N, Slater B, Silva MV. Consumo alimentar e excesso de peso de adolescentes de Piracicaba, São Paulo. Rev Nut. 2007;20(5):449-59.

3. Toral N, Conti MA, Slater B. A alimentação saudável na ótica dos adolescentes: percepções e barreiras à sua implementação e características esperadas em materiais educativos. Cad Saúde Pública. 2009;25(11):2386-94.

4. Brasil. Instituto Brasileiro de Geografia e Estatística-IBGE. Pesquisa Nacional de Saúde do Escolar 2009 [internet]. Rio de Janeiro; 2009 [cited 2014 Apr 10]. Available from: http://www.ibge.gov.br/home/estatistica/populacao/pense/pense.pdf

5. Azambuja APO, Netto-Oliveira ER, Azambuja MA, Oliveira AAB, Rinaldi W. Prevalência e fatores associados ao excesso de peso em escolares. Rev Baiana Saúde Pública. 2012;36(3):740-50.

6. Brasil. Ministério da Saúde. Secretaria de atenção à saúde. Protocolos do sistema de vigilância alimentar e nutricional-SISVAN [Internet]. Brasília; 2008. [cited 2013 Mar 10]. Available from: http://bvsms.saude.gov.br/bvs/publicacoes/protocolo_sistema_vigilancia_alimnetar.pdf

7. Currie C, Zanotti C, Morgan A, Currie D, Looze M, Roberts C et al., editors. Social determinants of health and well-being among young people - Health Behaviour in School-Aged Children (HBSC) study: international report from the 2009/2010 survey [internet]. Copenhagen: World Health Organization - WHO; Edinburg: University of Edinburgh, Child and Adolescent Health Research Unit - CAHRU; 2012 [cited 2014 Apr 10]. Available from: http://www.euro.who.int/_data/assets/pdf_file/0003/163857/Social-determinants-of-health-and-wellbeing-among-young-people.pdf

8. Nunes MMA, Figueiroa JN, Alves JGB. Excesso de peso, atividade física e hábitos alimentares entre adolescentes de diferentes classes econômicas em Campina Grande (PB). Rev Assoc Med Bras. 2007;53(2):130-4.

9. Levy RB, Castro IRR, Cardoso LO, Tavares LF, Sardinha LMV, Gomes FS, Costa A WN. Consumo e comportamento alimentar entre adolescentes brasileiros: Pesquisa Nacional de Saúde do Escolar (PeNSE). Ciênc Saúde Coletiva. 2010;14(2):3085-97.

10. Santos JS, Costa MCO, Sobrinho CLN, Silva MCM, Souza KEP, Melo BO. Perfil antropométrico e consumo alimentar de adolescentes de Teixeira de Freitas - Bahia. Rev Nutr. 2005;18(5):623-32.

11. Veiga GV, Sichieri R. Correlation in food intake between parents and adolescents depends on socioeconomic level. Nutr Res. 2006;26(10):517-23

12. Gomes FS. Frutas, legumes e verduras: recomendações técnicas versus constructos sociais. Rev Nutr. 2007;20(6):669-80.

13. Brasil. Instituto Brasileiro de Geografia e Estatística-IBGE. Pesquisa Nacional de Saúde do Escolar 2012 [internet]. Rio de Janeiro; 2012 [cited 2014 Apr 10]. Available from: http://www.ibge.gov.br/home/estatistica/populacao/pense/2012/pense_2012.pdf

14. Toral N, Slater B, Cintra IP, Fisberg M. Comportamento alimentar de adolescentes em relação ao consumo de frutas e verduras. Rev Nutr. 2006;19(3):331-40.

15. Feuz AS, Assis MAA, Passos MMCF. Métodos de inquéritos alimentares com abordagens do comportamento alimentar. In: Fisberg RM, Slater B, Marchioni DML, Martini LA. Inquéritos alimentares:métodos e bases científicos. Barueri: Manole; 2005. p. 32-70.

16. Brasil. Ministério da Saúde. Guia alimentar para a população brasileira:promovendo a alimentação saudável [internet]. Brasília; 2006 [cited 2013 Mar 20]. Available from: http://189.28.128.100/nutricao/docs/geral/guia_alimentar_conteudo.pdf.

17. Brasil. Instituto Brasileiro de Geografia e Estatística-IBGE. Pesquisa de Orçamentos Familiares 2008-2009: avaliação nutricional da disponibilidade domiciliar de alimentos no Brasil [internet]. Rio de Janeiro; 2010 [cited 2014 Apr 10]. Available from: http://www.ibge. gov.br/home/estatistica/populacao/condicaodevida/pof/2008_2009_analise_consumo/pofanalise_2008_2009.pdf

18. Mendes KL, Catão LP. Avaliação do consumo de frutas, legumes e verduras por adolescentes de formiga - MG e sua relação com fatores socioeconômicos. Alim Nutr. 2010;21(2):291-6.

19. Ciochetto CR, Orlandi SP, Vieira MFA. Consumo de frutas e vegetais em escolares da rede pública no Sul do Brasil. Arch Latinoam Nutr. 2012;62(2):172-8.

20. Reis LC, Correia IC, Mizutani ES. Estágios de mudança do comportamento para o consumo de frutas e hortaliças e sua relação com o perfil nutricional e dietético de universitários. Einstein. 2014;12(1):48-54. 
21. Santana JT, Furtado MB, Pinho L. Promoção do consumo de frutas e verduras em adolescentes: relato de experiência. Rev Enferm UFPE. 2014;8(1):213-9.

22. Steptoe A, Wijetunge S, Doherty S, Wardle J. Stages of change for dietary fat reduction: associations with food intake, decisional balance and motives for food choice. Health Educ J. 1996;55(1):108-22.

23. Veiga GV, Costa RS, Araújo MC, Souza AM, Bezerra IN, Barbosa FS, Sichieri R, Pereira RA. Inadequação do consumo de nutrientes entre adolescentes brasileiros. Rev Saúde Pública. 2013;47(S1):212s-21s.

24. Carvalho CMRG, Nogueira AMT, Teles JBM, Paz SMR, Sousa RML. Consumo alimentar de adolescentes matriculados em um colégio particular de Teresina, Piauí, Brasil. Rev Nutr. 2001;14(2):85-93.

25. Figueiredo IC, Jaime PC, Monteiro CA. Fatores associados ao consumo de frutas, legumes e verduras em adultos da cidade de São Paulo. Rev Saúde Pública. 2008;42(5):777-85.

26. Muniz LC, Zanini RV, Schneider BC, Tassitano RM, Feitosa WMN, Chica DAG. Prevalência e fatores associados ao consumo de frutas, legumes e verduras entre adolescentes de escolas públicas de Caruaru, PE. Ciênc Saúde Coletiva. 2013;18(2):393-404.

27. Currie C, Roberts C, Morgan A, Smith R, Settertobulte W, Samdal O. et al. Young people's health in context. Health Behaviour in School aged Children (HBSC) study: international report from the 2001/2002 survey [internet]. Genebra-WHO; 2004[cited 2013 Mar 20]. Available from: http://www.euro.who.int/_data/assets/pdf_file/0008/110231/e82923.pdf.

28. Prochaska JO, Di Clemente CC, Norcross JC. In search of how people change - applications to addictive behaviors. Am Psychol. 1992;47(9):1102-14.

29. Enes CC, Slater B. Obesidade na adolescência e seus principais fatores determinantes. Rev Bras Epidemiol. 2010;13(1):163-71.

30. Garcia GCB, Gambardella AMD, Frutuoso MFP. Estado nutricional e consumo alimentar de adolescentes de um centro de juventude da cidade de São Paulo. Rev Nutr. 2003;16(1):41-50. 